\title{
Emprego de uma nova emulsão de perfluorocarbonos (Oxygent MR) em circulação extracorpórea: estudo experimental em cães
}

Walter V. A. VICENTE* ${ }^{\star}$, William L. HOLMAN ${ }^{\star *}$, Russell D. SPRUELL ${ }^{\star *}$, Edward R. FERGUSON ${ }^{\star *}$, Janice J. CLYMER ${ }^{\star \star \star}$, C. Patric MURRAH ${ }^{\star \star}$, Albert D. PACIFICO**

RBCCV 44205-256

VICENTE, W. V. A.; HOLMAN, W. L.; SPRUELL, R. D.; FERGUSON, E. R.; CLYMER, J. J.; MURRAH, C. P.; PACIFICO, A. D. - Emprego de uma nova emulsão de perfluorocarbono (Oxygent MR) em circulação extracorpórea: estudo experimental em cães. Rev. Bras. Cir. Cardiovasc., 10 (1): 34-42, 1995.

RESUMO: Testou-se uma nova emulsão de perfluorocarbonos (Oxygent ${ }^{M R}$, Alliance Pharmaceutical, San Diego, CA 92121, EUA) em circulaçāo extracorpórea (CEC) com hipotermia de $32^{\circ} \mathrm{C}$ e hematócrito de $12 \%$. Estudaram-se 42 cães, 12 dos quais não receberam a droga e serviram de controle (Grupo 1), enquanto os demais constituíram 3 grupos de 10 animais cada, tratados com doses de Oxygent de 1,5 ml/kg (Grupo2), $3 \mathrm{ml} / \mathrm{kg}$ (Grupo 3 ) e $6 \mathrm{ml} / \mathrm{kg}$ (Grupo 4) as quais geraram fluorocritos de, respectivamente, $1 \%, 2 \%$ e $3 \%$. Foram analisadas variáveis do metabolismo do oxigênio $\left(\mathrm{O}_{2}\right)$ em 6 diferentes fluxos de perfusão $(\mathrm{Q})$, ordenados ao acaso. Reaqueceram-se os cães, interrompeu-se a CEC e acompanharam-se os animais por 1 hora. Diferenças intergrupos foram analisadas pelo teste das médias dos quadrados mínimos e pelo teste de Duncan, considerando-se significantes os valores de $p<0,05$. Embora algo maiores no Grupo 4, a pressão parcial bem como o conteúdo arterial de oxigênio $\left(\mathrm{C}_{\mathrm{a}} \mathrm{O}_{2}\right)$ foram estatisticamente semelhantes ao grupo controle. A pressão parcial de $\mathrm{O}_{2}$ no sangue venoso misto $\left(\mathrm{P}_{\mathrm{v}} \mathrm{O}_{2}\right)$ do Grupo 4, em função do $Q$, mostrou-se significativamente maior $(p<0,03)$ que a do grupo controle, conquanto isto não tenha se repetido ao ser analisada em função da oferta tecidual de $\mathrm{O}_{2}$. Também não houve diferença quanto ao consumo de $\mathrm{O}_{2}$ total e dissolvido, nem quanto aos gradientes sistêmico e miocárdico de lactato, porém a maior dose da emulsão (Grupo 4) expressa efeito dose-dependente benéfico, ainda que de natureza especulativa, do Oxygent sobre o metabolismo do oxigênio.

DESCRITORES: Perfluorocarbonos, em circulação extracorpórea. Sangue, metabolismo. Circulação extracorpórea.

\section{INTRODUÇÃO}

Em decorrência dos novos riscos infecciosos das transfusões, reacendeu-se o interesse pelo emprego de substitutos sangüíneos capazes de transportar grandes quantidades de oxigênio $\left(\mathrm{O}_{2}\right)^{4,29}$ como as soluções de hemoglobina ${ }^{8}$ e as emulsões de perfluorocarbonos (PFC) ${ }^{23}$. Por outro lado, estas últimas, recentemente, tornaram-se mais biocompatíveis, puras e concentradas, capazes de dissolver 4 a 5 vezes mais $\mathrm{O}_{2}$ que as anteriormente disponiveis ${ }^{17,20,23}$. Como a vida média intravascular desses novos PFC continua sendo de poucas horas, tais produtos são ainda mais adequados à paliação aguda

Trabalho realizado durante estágio de pós-doutorado nos EUA, financiado pelo Conselho Nacional de Desenvolvimento Científico e Tecnológico (CNPq).

Apresentado ao $22^{2}$ Congresso Nacional de Cirurgia Cardíaca. Brasília, DF, 30 de março de a $1^{2}$ de abril, 1995.

* Da Disciplina de Cirurgia Torácica e Cardiovascular - Depto. de Cirurgia da Faculdade de Medicina de Ribeirão Preto da Universidade de São Paulo.

** Division of Cardiothoracic Surgery. Univesity of Alabama at Birmingham and Birmingham V.A. Medical Center. Birmingham, AL, EUA.

$* \star *$ Alliance Pharmaceutical Corp. San Diego, CA, EUA.

Endereço para correspondência: Walter V. A. Vicente. Depto. de Cirurgia. H. C. Ribeirāo Preto - Campus da USP. Ribeirão Preto, SP, Brasil. CEP: 14049-900. Tel.: (016) 633.0836. Fax: (016) 633.1144. 
VICENTE, W. V. A.; HOLMAN, W. L.; SPRUELL, R. D.; FERGUSON, E. R.; CLYMER, J. J.; MURRAH, C. P.; PACIFICO, A. D. Emprego de uma nova emulsão de perfluorocarbono (Oxygent ${ }^{M R}$ ) em circulaçăo extracorpórea: estudo experimental em cães. Rev. Bras. Cir. Cardiovasc., 10 (1): 34-42, 1995.

e transitória de redução do transporte do $\mathrm{O}_{2}$, v.g., durante CEC com hemodiluição podendo, inclusive, até otimizar as sangrias pré-CEC, para autotransfusão posterior 13

Testou-se neste trabalho a hipótese de que uma nova emulsão de perfluorocarbonos (Oxygent MR [AF0142], Alliance Pharmaceutical, San Diego, CA, EUA) aumente a fração de $\mathrm{O}_{2}$ dissolvido no sangue in vivo. $\mathrm{O}$ objetivo foi de avaliar os efeitos do produto sobre o metabolismo do $\mathrm{O}_{2}$ em cães submetidos à CEC conduzida com hematócrito de $12 \%$.

\section{MATERIAL E MÉTODOS}

Cães mestiços adultos, de ambos os sexos ( $\mathrm{N}$ $=42$ ), com $20 \mathrm{~kg}$ a $39 \mathrm{~kg}$ (média $=28 \mathrm{~kg}$ ) de peso, foram distribuídos em 4 grupos. O Grupo 1 (12 cães) não recebeu a droga e serviu de controle; os outros 3 (de 10 animais cada) receberam $1,5 \mathrm{ml} / \mathrm{kg}$ (Grupo 2), $3 \mathrm{ml} / \mathrm{kg}$ (Grupo 3) e $6 \mathrm{ml} / \mathrm{kg}$ (Grupo 4) de Oxygent. Os animais foram anestesiados com pentobarbital sódico ( $30 \mathrm{mg} / \mathrm{kg} \mathrm{EV}$ ), posicionados sobre colchão térmico, curarizados com brometo de pancurônio $(0,3 \mathrm{mg} / \mathrm{kg} \mathrm{EV})$, intubados e ventilados.

Inseriram-se um termômetro esofágico e eletrodos nas patas, canularam-se as veias jugulares externas e as artérias carótida comum esquerda e femoral direita e, por laparotomia e esternotomia medianas, realizaram-se esplenectomia e cateterismo do seio coronário ${ }^{13}$, átrio esquerdo e artéria pulmonar. Após heparinização sistêmica $(3 \mathrm{mg} / \mathrm{kg}$ EV), induziu-se hemodiluição ${ }^{14}$ por sangria arterial, concomitante à reposição volêmica $\mathrm{EV}$, com solução composta por $100 \mathrm{ml}$ de cloreto de sódio a $0,9 \%$, $5 \mathrm{mEq}$ de cloreto de potássio e $50 \mathrm{mEq}$ de bicarbonato de sódio, a $38^{\circ} \mathrm{C}$, de modo a manter constante a pressão atrial esquerda (PAE). A hemodiluição, com duração de $15 \mathrm{~min}$ a $25 \mathrm{~min}$, foi interrompida ao atingir-se hematócrito de $15 \%$.

Canulou-se o átrio direito, estabeleceu-se CEC normotérmica átrio direito-femoral com fluxo de perfusão de $2,0 \mathrm{l} / \mathrm{min} / \mathrm{m}^{2}$, e administrou-se Oxygent pelo reservatório do oxigenador. Descomprimiramse as câmaras esquerdas, resfriaram-se os animais até $32^{\circ} \mathrm{C}$ e ajustou-se o hematócrito em $12 \%$. Os cães foram, então, perfundidos com 6 fluxos diferentes $\left(0,25 ; 0,5 ; 1,0 ; 1,5 ; 2,0\right.$ e $\left.3,0 \mathrm{l} / \mathrm{min} / \mathrm{m}^{2}\right)$, ordenados ao acaso. Cinco minutos após o início de cada fluxo, colhiam-se amostras de sangue e, completada a série, reaqueciam-se os cães e interrompia-se a CEC. No pós-CEC, a PAE foi mantida pela infusão da mesma solução utilizada na hemodiluição. Os animais foram observados por $1 \mathrm{~h}$ e sacrificados com cloreto de potássio EV. No pós-CEC, foram declarados mortos aqueles cuja pressão arterial sistólica se reduzisse a menos de $20 \mathrm{mmHg}$. A CEC foi conduzida com oxigenador Maxima (Medtronic), bomba de roletes (modelo 5000 , Sarns) e perfusato cristalóide. A relação entre os fluxos de $\mathrm{O}_{2}$ e de sangue foi de 2/1. Para evitar queda da $\mathrm{P}_{\mathrm{a}} \mathrm{CO}_{2}$ abaixo de $30 \mathrm{mmHg}$, adicionou-se gás carbônico com fluxo de até $500 \mathrm{ml} / \mathrm{min}$.

GRÁFICO 1

(A E B) - HEMATÓCRITO (Hb) E CONCENTRAÇÃO DE HEMOGLOBINA (Hb) DAS AMOSTRAS DE 1 A 15 (MÉDIA \pm ERRO PADRĀO). NOTAR A SEMELHANÇA ENTRE OS GRUPOS APÓS A HEMODILUIÇÃO NORMOVOLÊMICA (AMOSTRA 2), A QUEDA ADICIONAL DE AMBAS AS VARIÁVEIS COM O INICIO DA CEC (AMOSTRA 3), BEM COMO OS VALORES ESTÁVEIS, EM TORNO DE $12 \%$ E 4 g/dI DURANTE O PERIODO DE CEC A $32^{\circ} \mathrm{C}$ (AMOSTRAS DE 4 A 9).

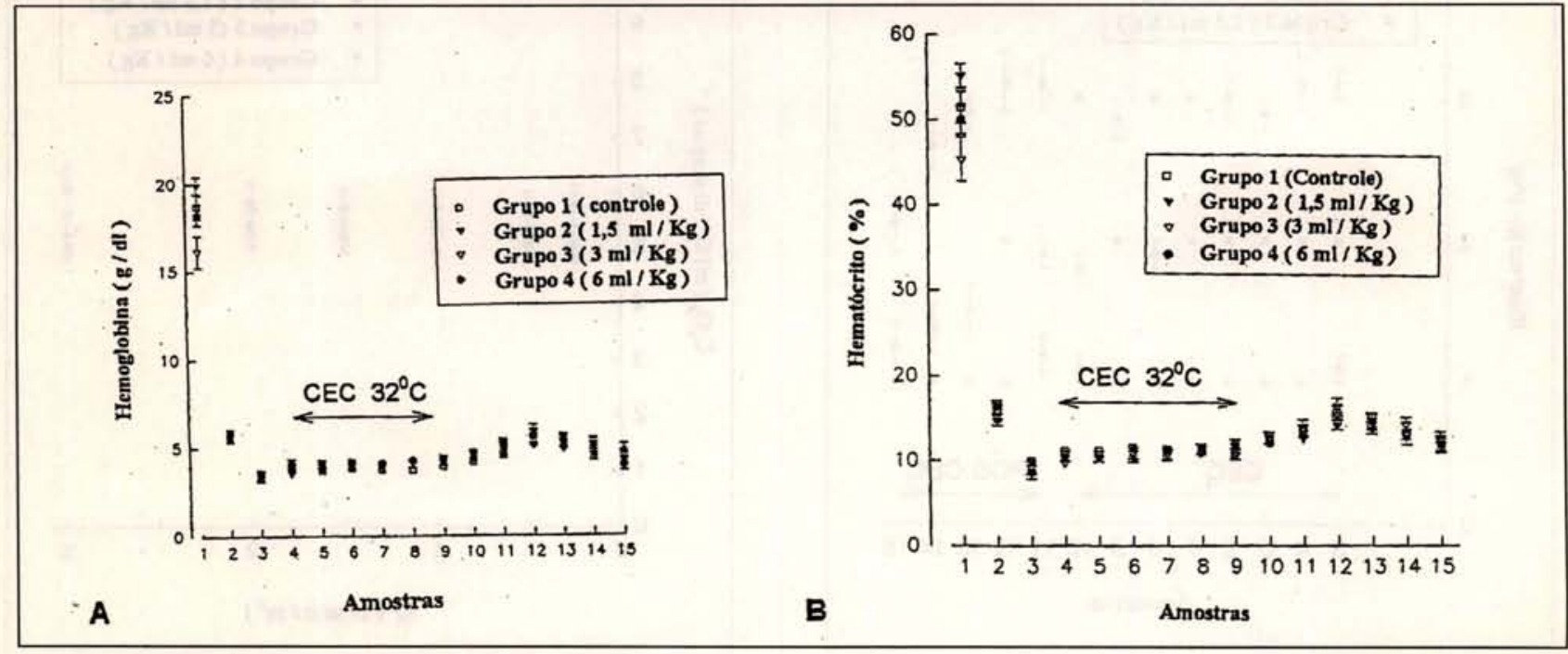


VICENTE, W. V. A.; HOLMAN, W. L.; SPRUELL, R. D.; FERGUSON, E. R.; CLYMER, J. J.; MURRAH, C. P.; PACIFICO, A. D. Emprego de uma nova emulsão de perfluorocarbono (Oxygent MR) em circulação extracorpórea: estudo experimental em cães. Rev. Bras. Cir. Cardiovasc., 10 (1): 34-42, 1995.

A pressão média e fásica das artérias pulmonar e carótida, bem como a pressão dos átrios e o eletrocardiograma (transdutores e amplificadores da Gould Electronics), foram gerenciados com o programa CODAS (Dataq Inc.), num computador 386. Colheram-se amostras simultâneas de sangue da artéria pulmonar (da cânula venosa, durante CEC), do seio coronário e da artéria carótida, numeradas conforme segue: antes (1) e depois (2) da hemodiluição normovolêmica; 5 min após o início da CEC normotérmica (3); em CEC hipotérmica, 5 min após - início de cada um dos 6 fluxos de perfusão (4 a 9); antes (10) e imediatamente após (11) o término da CEC; e aos 15, 30, 45 e 60 min subseqüentes (12 a 15). O hematócrito e o fluorocrito foram quantificados em tubos de micro-hematócrito (centrífuga $\mathrm{MB}$, International Equipment $\mathrm{Co}$.). A concentração $(\mathrm{g} / \mathrm{dl})$ e a saturação da hemoglobina do sangue venoso misto e $\circ \mathrm{pH}, \mathrm{pCO}_{2}, \mathrm{pO}_{2}$ e bicarbonato arterial e venoso misto foram medidos (CO Oxímetro modelo 282, Instrumentation Laboratories e analizador de gases Ciba-Corning, modelo 238), sem correção para a temperatura corpórea. $\mathrm{O}$ conteúdo de $\mathrm{O}_{2}$ do sangue arterial $\left(\mathrm{C}_{\mathrm{a}} \mathrm{O}_{2}\right)$, do venoso misto $\left(\mathrm{C}_{\mathrm{v}} \mathrm{O}_{2}\right)$ e do seio coronário $\left(\mathrm{C}_{\mathrm{sc}} \mathrm{O}_{2}\right)$ foi determinado em ml/dl (Lex $\mathrm{O}_{2}$ Con, Lexington Instruments Corp.) e calculou-se o conteúdo de $\mathrm{O}_{2}$ dissolvido no sangue arterial, venoso misto e do seio coronário ao subtrair-se do $\mathrm{C}_{\mathrm{a}} \mathrm{O}_{2}$, do $\mathrm{C}_{\mathrm{v}} \mathrm{O}_{2}$ e do $\mathrm{C}_{\mathrm{sc}} \mathrm{O}_{2}$, o volume de $\mathrm{O}_{2}$ quelado à hemoglobina $\left(\mathrm{C}_{\mathrm{Hb}} \mathrm{O}_{2}\right)$, representa-

GRÁFICO 2

FLUOROCRITO (MÉDIA \pm ERRO PADRĀO) DAS AMOSTRAS DE 3 (5 MIN APÓS O INICIO DA CEC) A 15 (1H PÓS-CEC). NOTAR A ESTABILIDADE DO FLUOROCRITO DURANTE A CEC EA TENDENNCIA À QUEDA NO PÓS-CEC.

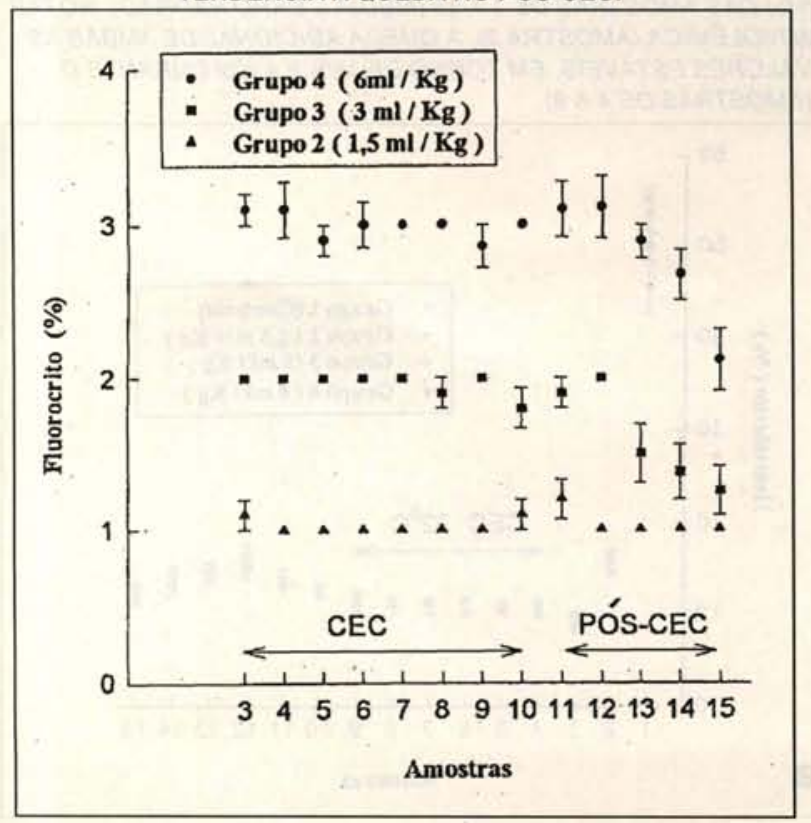

do pela equação: $\mathrm{C}_{\mathrm{Hb}} \mathrm{O}_{2}=\mathrm{Hb} \times$ Sat $\times 1,34$. Determinaram-se, com base na equação de Fick, em $\mathrm{ml} /$ $\mathrm{min} / \mathrm{m}^{2}$, a oferta $\left(\mathrm{DO}_{2}\right) \cdot$ e o consumo de $\mathrm{O}^{2}\left(\mathrm{VO}_{2}\right)$ nos 6 fluxos testados, bem como a oferta $\left(\mathrm{DO}_{2}\right.$ dissolvido) e o consumo $\left(\mathrm{VO}_{2}\right.$ dissolvido) de $\mathrm{O}_{2}$ transportado em dissolução física. O lactado sérico foi dosado (analisador Cobas Fara II, Roche Diagnostic Systems) em $\mathrm{mMol} / \mathrm{ml}$ e calcularam-se seus gradientes sistêmico e miocárdico.

Os resultados foram dispostos em gráficos (programa Sigma Plot, versões "DOS" 5.0 e "windows" 1.02) e submetidos a análise estatística com os testes das médias dos quadrados mínimos e de Duncan, contidos no programa SAS-PC, versão 6.03 (SAS Institute, Inc.), considerando-se significantes os valores de $p<0,05$.

\section{RESULTADOS}

Ao se aplicarem os 6 fluxos de perfusão, o Hto e a $\mathrm{Hb}$ dos 4 grupos foram semelhantes, ao redor de $12 \%$ e $4 \mathrm{mg} / \mathrm{dl}$, respectivamente (Gráfico 1), e o fluorocrito estável (Gráfico 2), de cerca de $3 \%$ no Grupo $4,2 \%$ no Grupo 3 e $1 \%$ no Grupo 2. Nas 3 últimas amostras pós-CEC, o fluorocrito decresceu com certa rapidez, apenas nos Grupos 3 e 4. O $\mathrm{C}_{\mathrm{a}} \mathrm{O}_{2}$ (Gráfico 3) e $0 \mathrm{C}_{\mathrm{v}} \mathrm{O}_{2}$ (Gráfico 4) também foram considerados similares nos 4 grupos, apesar

\section{GRÁFICO 3}

RELAÇĀO ENTRE O CONTEÜDO ARTERIAL DE OXIGÊNIO $\left(\mathrm{C}_{\mathrm{a}} \mathrm{O}_{2}\right)$ E O FLUXO DE PERFUSĀO (Q), DURANTE CEC HIPOTÉRMICA, A $32^{\circ} \mathrm{C}$. NĀO OBSTANTE OS VALORES LIGEIRAMENTE SUPERIORES DO GRUPO 4, NĀO HOUVE DIFERENÇA ESTATISTICAMENTE SIGNIFICANTE.

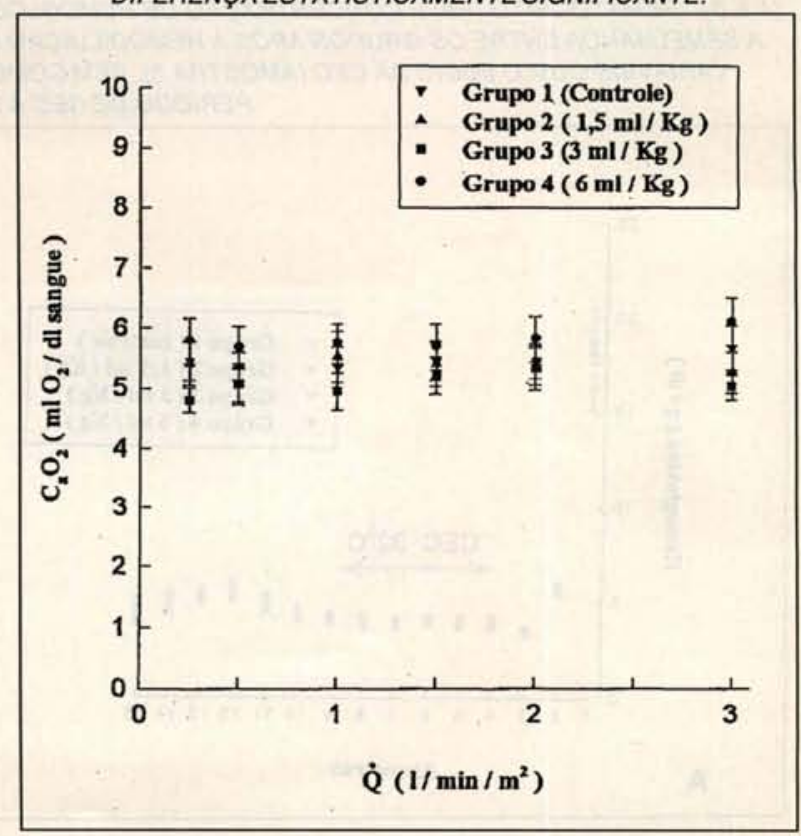


VICENTE, W. V. A.; HOLMAN, W. L.; SPRUELL, R. D.; FERGUSON, E. R.; CLYMER, J. J.; MURRAH, C. P.; PACIFICO, A. D. Emprego de uma nova emulsão de perfluorocarbono (Oxygent ${ }^{\text {MRa) }}$ ) em circulação extracorpórea: estudo experimental em cães. Rev. Bras. Cir. Cardiovasc., 10 (1): 34-42, 1995.

GRÁFICO 4

DURANTE CEC HIPOTÉRMICA, A $32^{\circ} \mathrm{C}, O$ CONTEÚDO DE OXIGÊNIO DO SANGUE VENOSO MISTO $\left(\mathrm{C}_{1} \mathrm{O}_{2}\right)$ DOS CĀES DOS 4 GRUPOS (MÉDIA \pm ERRO PADRÃO) GUARDOU RELAÇÃO DIRETA COM O FLUXO DE PERFUSĀO (Q). EMBORA O GRUPO 4 SE SOBRESSAIA DOS DEMAIS, AS DIFERENÇAS NÄO FORAM ESTATISTICAMENTE SIGNIFICANTES.

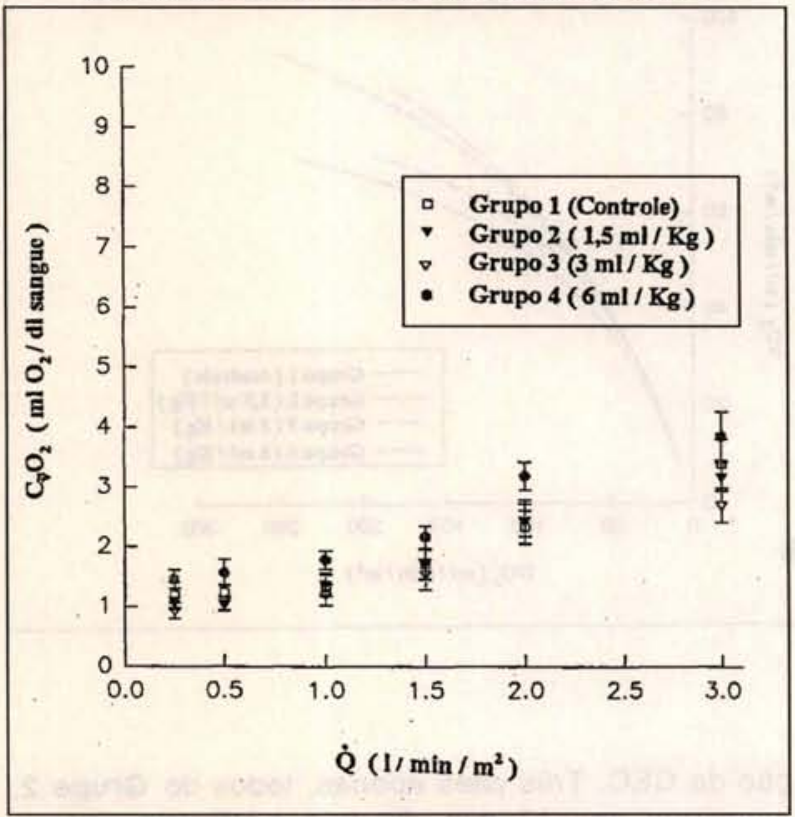

de ligeiramente maiores no Grupo 4. O mesmo se deu quanto à $\mathrm{P}_{\mathrm{a}} \mathrm{O}_{2}$, nunca inferior a $500 \mathrm{mmHg}$ (Gráfico 5). O Grupo 4, porém, caracterizou-se por $\mathrm{P}_{\mathrm{v}} \mathrm{O}_{2}$ estatisticamente maior $(\mathrm{p}<0,03)$ que a do controle. Entretanto, isto se verificou apenas ao

\section{GRÁFICO 5}

PRESSÃO PARCIAL DE OXIGÊNIO NO SANGUE ARTERIAL

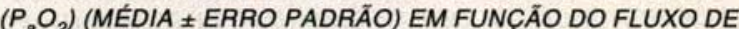
PERFUSÃO (Q), DURANTE CEC, A $32^{\circ} \mathrm{C}$. ATENTAR PARA OS VALORES ELEVADOS, PRÓXIMOS DE $600 \mathrm{mmHg}$, NOS 4 GRUPOS. APESAR DA $\mathrm{P}_{\mathrm{a}} \mathrm{O}_{2}$ SEMPRE MAIS ELEVADA NO GRUPO 4 E MENOS NÖ 2, NĀO HOUVE DIFERENÇAS ESTATISTICAMENTE SIGNIFICANTES.

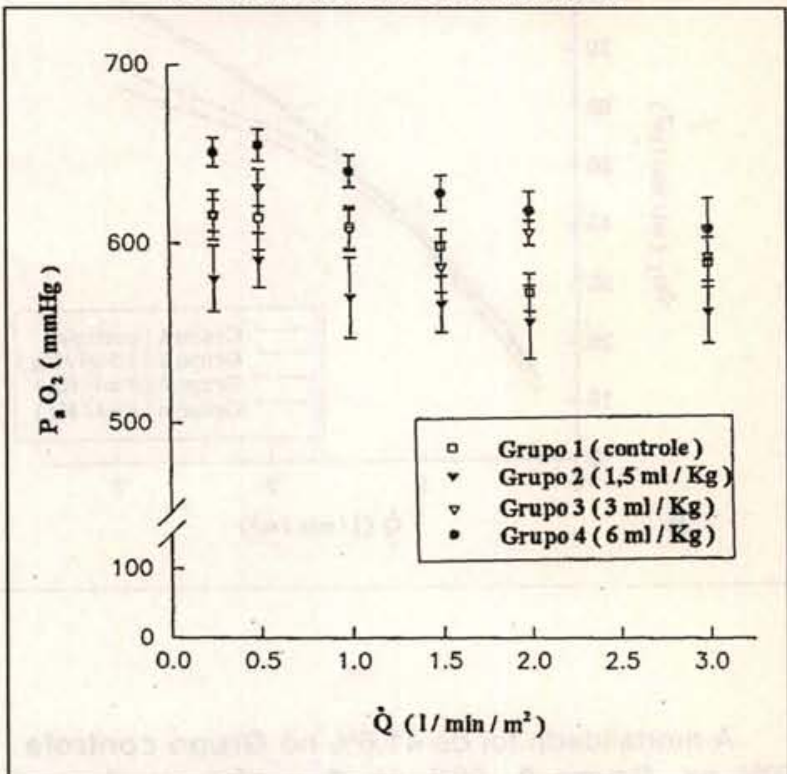

analisá-la em função do $\mathrm{Q}$, mas não da $\mathrm{DO}_{2}$ (Gráfico 6). Foram, ainda, semelhantes nos 4 grupos, tanto $\circ \mathrm{VO}_{2}$ (Gráfico 7), como $\circ \mathrm{VO}_{2}$ dissolvido (Gráfico 8) e os gradientes sistêmico e miocárdico de lactato (Gráfico 9).

\section{GRÁFICO 6 (A e B)}

REGRESSÃO DA PRESSÃO PARCIAL DE OXIGÊNIO DO SANGUE VENOSO MISTO $\left(P_{V} \mathrm{O}_{2}\right)$ EM FUNÇÃO TANTO DO FLUXO DE PERFUSÃO (Q) COMO DA OFERTA TECIDUAL DE OXIGÊNIO (DO,), DURANTE CEC, A $32^{\circ} \mathrm{C}$. OBSERVE-SE QUE AS CURVAS DOS GRUPOS QUE RECEBERAM OXYGENT PROJETARAM-SE SEMPRE MAIS ELEVADAS, DENOTANDO MAIOR $P_{y} \mathrm{O}_{2}$ EM FUNÇÃO QUER DO Q QUER DA DO ${ }_{2}$; ESSA DIFERENCA FOI SIGNIFICANTE (P<0.03) APENAS ENTRE OS TRACADOS DOS GRUPOS 4 E 1 , EM FUNCCĀO DO Q. AS BARRAS HORIZONTAIS CORRESPONDEM A VALORES DE $P_{v} \mathrm{O}_{2}$ ABAIXO DOS QUAIS ACREDITA-SE QUE SURJA HIPÓXIA TECIDUAL.
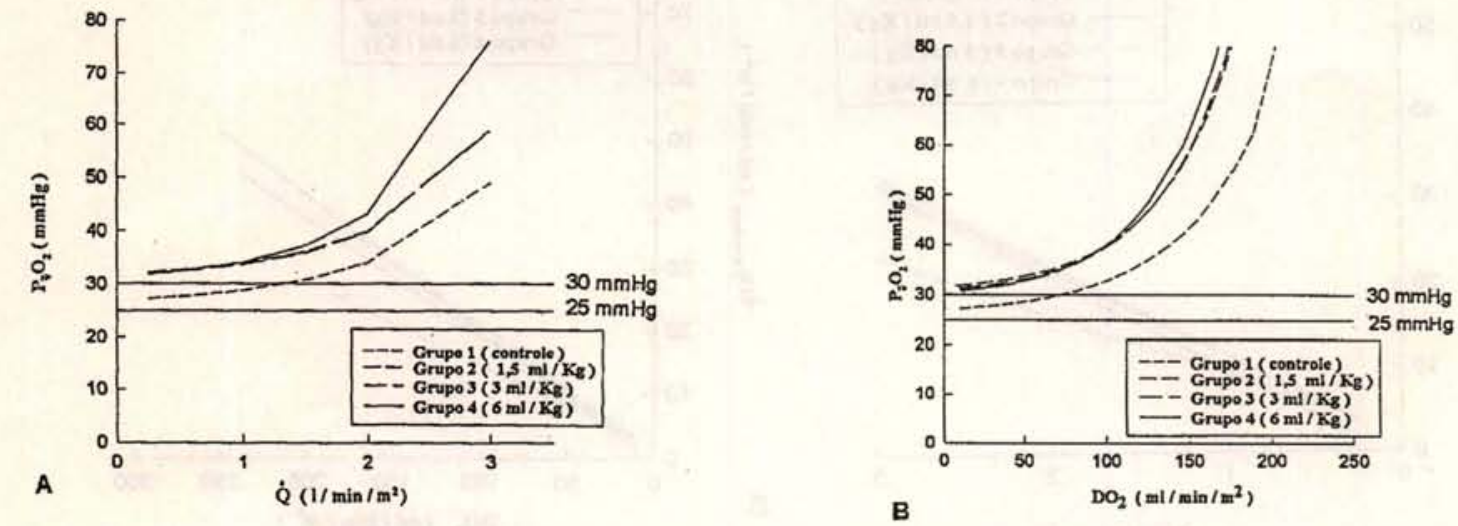
VICENTE, W. V. A.; HOLMAN, W. L.; SPRUELL, R. D.; FERGUSON, E. R.; CLYMER, J. J.; MURRAH, C. P.; PACIFICO, A. D. Emprego de uma nova emulsão de perfluorocarbono (Oxygent MR) em circulação extracorpórea: estudo experimental em cães. Rev. Bras. Cir. Cardiovasc., 10 (1): 34-42, 1995.

\section{GRÁFICO 7 (A e B)}

DURANTE CEC, A $32^{\circ} \mathrm{C}$, A REGRESSÃO DO CONSUMO DE OXIGÊNIO (VO ${ }_{2}$ ) EM FUNÇĀO TANTO DO FLUXO DE PERFUSĀO (Q) COMO DA OFERTA TECIDUAL DE OXIGÊNIO (DO,) DESCREVEU HIPÉRBOLES RETANGULARES ESTATISTICAMENTE SEMELHANTES, APESAR DE SE ESBOÇAR CERTA DIVERGÊNCIA ENTRE AS CURVAS COM O AUMENTO DO Q E DA DO ${ }_{2}$.

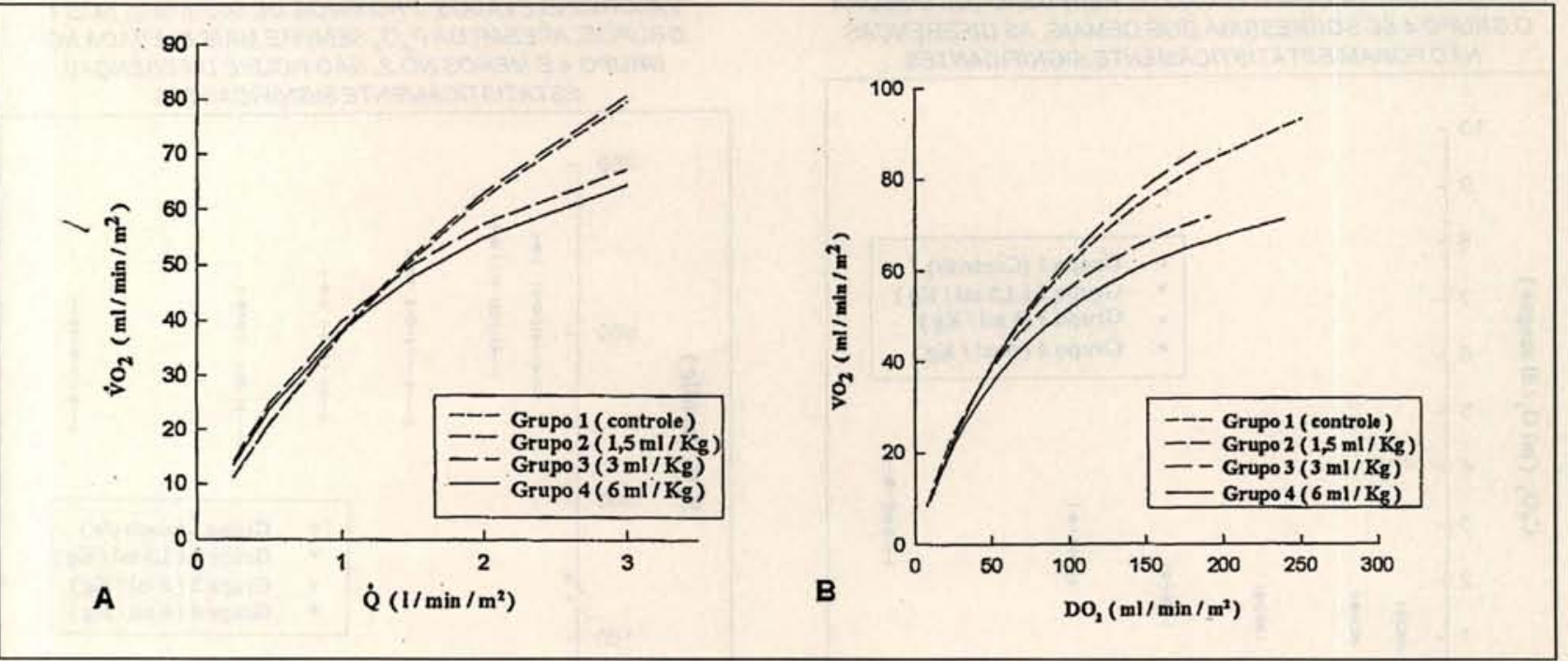

A mortalidade foi de $41,6 \%$ no Grupo controle, $50 \%$ no Grupo 2, $20 \%$ no 3 e $10 \%$ no 4 (que recebeu a maior dose de Oxygent), mas tais diferenças não foram estatisticamente significantes. A morte de quase todos os animais ocorreu por hipotensão arterial com grande elevação da PAE e acentuada distensão cardíaca, logo após a interrup- ção da CEC. Três cães apenas, todos do Grupo 2, morreram aos $17 \mathrm{~min}, 35 \mathrm{~min}$ e $40 \mathrm{~min}$ após o término da CEC, com o mesmo quadro clínico. Nestes, bem como nos que sobreviveram até ao final do período de observação, notou-se progressão do edema tecidual que todos os cães dos 4 grupos apresentaram no decorrer da CEC.

\section{GRÁFICO 8 ( $A$ e B)}

REGRESSÃO DO CONSUMO DE OXIGÊNIO DISSOL VIDO (VO TECIDUAL DE OXIGÊNIO $\left(D_{2}\right)$, DURANTE CEC, A $32^{\circ} \mathrm{C}$. NÄO OBSTANTE A NITIDA DIFERENÇA DE INCLINAÇÃO ENTRE AS RETAS DOS GRUPOS QUE RECEBERAM OXYGENT E O CONTROLE, NÄO HOUVE DIFERENÇA ESTATISTICAMENTE SIGNIFICANTE.
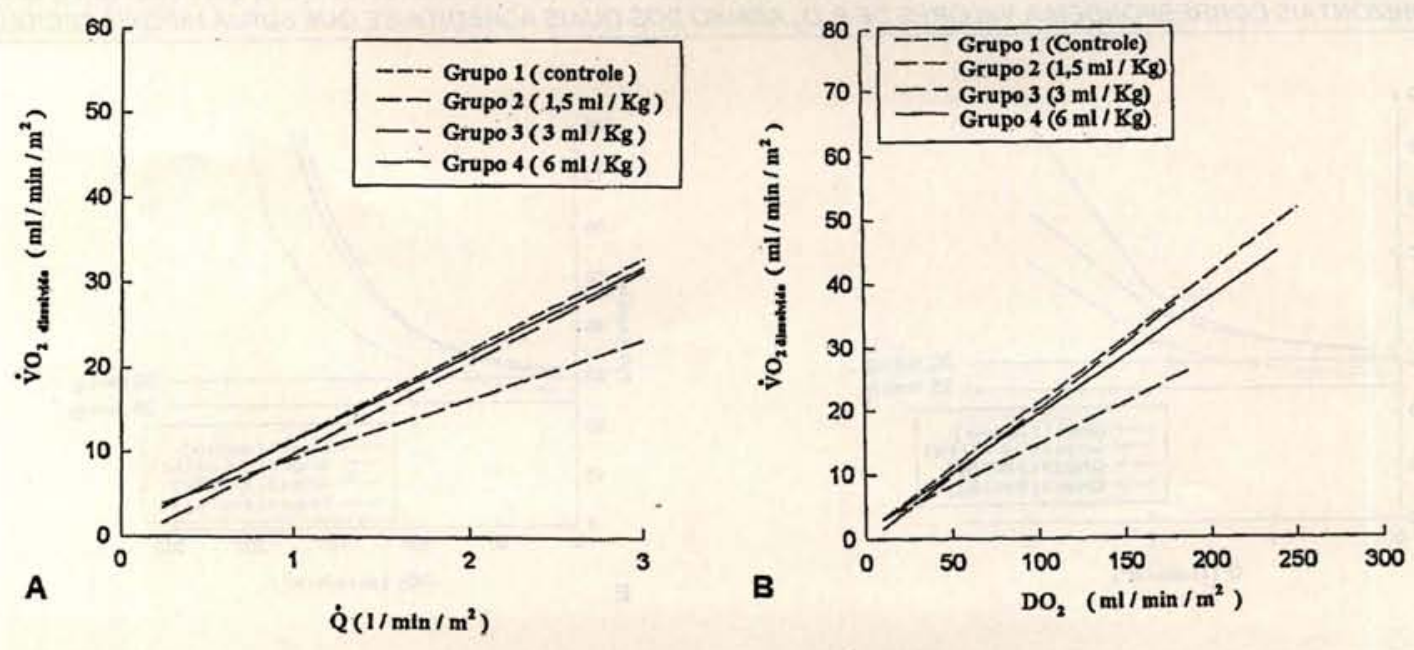
VICENTE, W. V. A.; HOLMAN, W. L.; SPRUELL, R. D.; FERGUSON, E. R.; CLYMER, J. J.; MURRAH, C. P.; PACIFICO, A. D. Emprego de uma nova emulsão de perfluorocarbono (Oxygent ${ }^{\mathrm{MR}}$ ) em circulação extracorpórea: estudo experimental em cäes. Rev. Bras. Cir. Cardiovasc., 10 (1): 34-42, 1995.

GRÁFICO 9 (A e B)

RETAS DE REGRESSÃO DOS GRADIENTES SISTÊMICO E MIOCÁRDICO DE LACTATO, EM FUNCCÃO DO FLUXO DE PERFUSÃO (Q), DURANTE CEC, A $32^{\circ} \mathrm{C}$. NOTAR A INVERSÄO DOS GRADIENTES, DE NEGATIVOS PARA POSITIVOS Ȧ MEDIDA QUE AUMENTA O Q. NĀO OCORRERAM DIFERENÇAS SIGNIFICATIVAS.

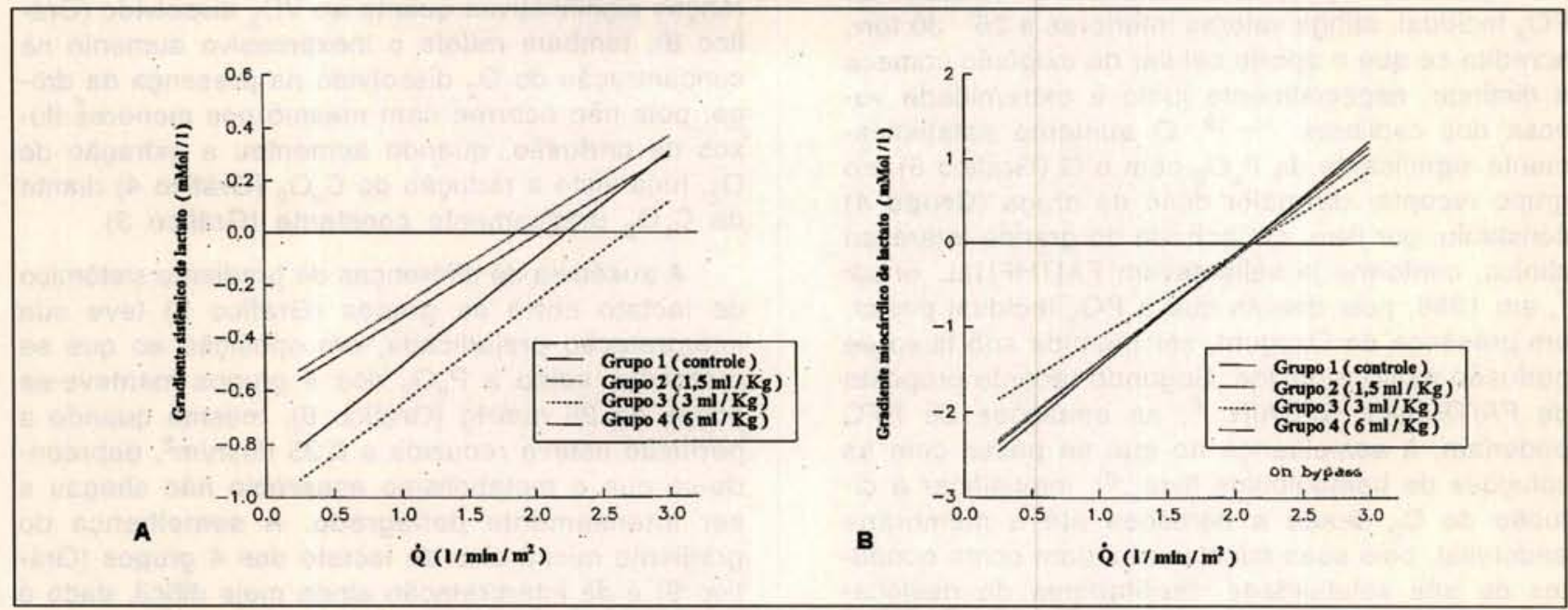

\section{COMENTÁRIOS}

Esta investigação, à semelhança de FOX et alii ${ }^{10}$, restringiu-se a um período de CEC hipotérmica no decurso do qual mantiveram-se constantes a temperatura esofágica e a Hb (Gráfico 1). Destarte, isolou-se a emulsão de PFC como única variável responsável pelas eventuais diferanças de $\mathrm{C}_{\mathrm{a}} \mathrm{O}_{2}$. Os altos níveis de $\mathrm{P}_{\mathrm{a}} \mathrm{O}_{2}$ durante a CEC (Gráfico 5 ) devem não só ter garantido completa saturação da hemoglobina como também favorecido a dissolução do $\mathrm{O}_{2}$ no Oxygent, uma vez que a massa de gás dissolvida pelos PFC aumenta de modo linear com a pressão parcial, acentua-se com a queda da temperatura e quase não varia com as mudanças de $\mathrm{pH}^{2}$. Esse cuidado, porém, não fora tomado em trabalhos anteriores, com outros PFC, estudados sob $\mathrm{P}_{\mathrm{a}} \mathrm{O}_{2}$ bem mais baixa $5,24,30$.

Incluíram-se no protocolo fluxos de perfusão diminutos, de até $0,25 \mathrm{l} / \mathrm{min} / \mathrm{m}^{2}$, na intenção de que a $\mathrm{DO}_{2}$ do grupo controle caísse a ponto de causar hipoxia tecidual. Para salientar a eficácia da droga, induziu-se anemia aguda intensa e hipotermia, com controle "alfa-stat" do $\mathrm{pH}$ sangüíneo, a fim de reduzir o consumo de $\mathrm{O}_{2}$ transportado pela hemoglobina ${ }^{31}, 12$, não obstante possa também ocorrer maior afinidade dos tecidos pelo $\mathrm{O}_{2}$ nessa situação ${ }^{18}$.

O Oxygent foi bem tolerado, mas a estabilidade do fluorocrito durante a CEC (Gráfico 2) constituiu achado intrigante, pois se sabe que as micelas contendo PFC são continuamente fagocitadas pelo sistema retículo-endotelial (SRE) 21, 22 . Possivel- mente, isso refletiu redução na atividade ou sobrecarga do SRE devido ao aparecimento de outros tipos de partículas circulantes, as quais teriam competido com as gotículas de Oxygent no processo de fagocitose. Por outro lado, a esplenectomia, realizada para permitir melhor controle da hemodiluição, pode, também, ter contribuído para a lenta remoção do produto, conquanto a queda relativamente rápida do fluorocrito no pós-CEC, nos Grupos 3 e 4, talvez tenha decorrido ou de maior captação pelo SRE, ou de aumento de permeabilidade capilar, grave o suficiente para permitir o extravazamento da emulsão para o interstício.

A ineficácia da droga em aumentar $0 \quad \mathrm{C}_{\mathrm{a}} \mathrm{O}_{2}$ (Gráfico 3) contrastou com estudos in vitro realizados no mesmo laboratório 9,12 , que demonstraram aumento significativo de transporte de oxigênio por um perfusato cristalóide quando acrescido de Oxygent. Esta contradição talvez se devesse à utilização, nos estudos in vitro de fluorocrito cerca de 4 vezes maior $(12,5 \%)$ que o do grupo que recebeu a maior dose $(3 \%)$ neste, in vivo (Gráfico 3$)$. Ainda que se procurasse exacerbar a proporcionalidade entre o fluorocrito e a $\mathrm{Hb}$ graças à intensa hemodiluição, pode-se conjecturar que os baixos fluorocritos empregados tenham gerado elevações de $\mathrm{C}_{\mathrm{a}} \mathrm{O}_{2}$ muito pequenas, abaixo do poder de discriminação do método de medição adotado ${ }^{25}$. Daí, talvez, ter sidc detectada discreta elevação do $\mathrm{C}_{\mathrm{a}} \mathrm{O}_{2}$, ainda que sem significância estatística, apenas no Grupo 4 (Gráfico 3). O mesmo pode ter sucedido com relação ao $\mathrm{C}_{\mathrm{v}} \mathrm{O}_{2}$, também pouco mais elevado no 
VICENTE, W. V. A.; HOLMAN, W. L.; SPRUELL, R. D.; FERGUSON, E. R.; CLYMER, J. J.; MURRAH, C. P.; PACIFICO, A. D. Emprego de uma nova emulsão de perfluorocarbono (Oxygent MR) em circulação extracorpórea: estudo experimental em cães. Rev. Bras. Cir. Cardiovasc., 10 (1): 34-42, 1995.

grupo tratado com a maior dose da emulsão (Gráfico 4).

Quando $\circ \mathrm{P}_{\mathrm{v}} \mathrm{O}_{2}$, considerado um indicador do $\mathrm{PO}_{2}$ tecidual, atinge valores inferiores a 25 - 30 torr, acredita-se que o aporte celular de oxigênio comece a diminuir, especialmente junto à extremidade venosa dos capilares 16,19 . O aumento estatisticamente significante da $\mathrm{P}_{\mathrm{v}} \mathrm{O}_{2}$ com $\circ \mathrm{Q}$ (Gráfico 6), no grupo receptor da maior dose da droga (Grupo 4) constituiu, por isso, um achado de grande interesse clínico, conforme já salientavam FAITHFULL et alii ${ }^{7}$, em 1986, pois denota que a $\mathrm{PO}_{2}$ tecidual possa, em presença de Oxygent, ser mantida sob fluxo de perfusão muito reduzido. Segundo recente proposta de FAITHFULL \& CAIN 6 , as emulsões de PFC poderiam, à semelhança do que se passa com as soluções de hemoglobina livre ${ }^{26}$, intensificar a difusão do $\mathrm{O}_{2}$ desde a hemácea até a membrana endotelial, pois suas micelas atuariam como condutos de alta solubilidade, facilitadores do deslocamento desse gás através do plasma, um reconhecido mau solvente do $\mathrm{O}_{2}{ }^{15}$. Esse mecanismo seria análogo à travessia mais expedita de um riacho, ao saltar sobre pedras expostas acima da linha d'água 22 . De acordo com a inovadora concepção reológica da circulação proposta por ZAKHAROV ${ }^{32}$, é possível que as partículas da emulsão de Oxygent, cerca de 35 vezes menores que as dos eritrócitos ${ }^{1}$, além de disporem de maior superfície de troca em proporção ao volume corpuscular, o que facilita a difusão do $\mathrm{O}_{2}$, se distribuam na microcirculação de modo a formar uma interface laminar dinâmica entre as hemácias e a parede capilar propícia à intermediação nas trocas gasosas com os tecidos. Embora a discreta elevação do $\mathrm{C}_{\mathrm{v}} \mathrm{O}_{2}$ detectada no Grupo 4 possa, outrosim, explicar o aumento da $\mathrm{P}_{v} \mathrm{O}_{2}$ neste grupo, nos outros 2 grupos tratados; entretanto, $\circ \mathrm{C}_{\mathrm{v}} \mathrm{O}_{2}$ esteve bem próximo ao do Grupo controle (Gráfico 4). Apesar de não ter havido diferença estatística ao avaliar-se a $\mathrm{P}_{\mathrm{v}} \mathrm{O}_{2}$ em função da $\mathrm{DO}_{2}$ (Gráfico 6), repetiram-se resultados nitidamente melhores nos grupos tratados, cujas curvas de regressão, curiosamente, quase se superpuseram.

$\mathrm{Na}$ presença de Oxygent, esperava-se aumento do $\mathrm{VO}_{2}$, particularmente nos menores fluxos de perfusão. Tal fato, no entanto, não ocorreu (Gráfico 7), certamente porque não houve aumento no $\mathrm{C}_{\mathrm{a}} \mathrm{O}_{2}$ (Gráfico 3). Representou-se $\circ \mathrm{VO}_{2}$ versus a $\mathrm{DO}_{2}$ (Gráfico 7) para tentar corroborar a teoria da facilitação das trocas gasosas ao nível da microcirculação já referida 6 pois, eventualmente, poder-se-ia constatar aumento de $\mathrm{VO}_{2}$ em função da $\mathrm{DO}_{2}$, especialmente nos menores fluxos de perfusão, o que, entretanto, não ocorreu. A ausência de diferenças significativas quanto ao $\mathrm{VO}_{2}$ dissolvido (Gráfico 8), também reflete o inexpressivo aumento na concentração de $\mathrm{O}_{2}$ dissolvido na presença da droga, pois não ocorreu nem mesmo nos menores fluxos de perfusão, quando aumentou a extração de $\mathrm{O}_{2}$, haja visto a redução do $\mathrm{C}_{\mathrm{v}} \mathrm{O}_{2}$ (Gráfico 4) diante da $\mathrm{C}_{\mathrm{a}} \mathrm{O}_{2}$ praticamente constante (Gráfico 3 ).

A ausência de diferenças de gradiente sistêmico de lactato entre os grupos (Gráfico 9) teve sua interpretação prejudicada; em oposição ao que se pretendia, como a $\mathrm{P}_{\mathrm{v}} \mathrm{O}_{2}$ dos 4 grupos manteve-se acima de $25 \mathrm{mmHg}$ (Gráfico 6), mesmo quando a perfusão esteve reduzida a $0,25 \mathrm{l} / \mathrm{min} / \mathrm{m}^{2}$, depreende-se que o metabolismo anaeróbio não chegou a ser intensamente deflagrado. A semelhança do gradiente miocárdico de lactato dos 4 grupos (Gráfico 9) é de interpretação ainda mais difícil, dado o grande número de fatores que influenciam o metabolismo cardíaco deste íon 27 .

A redução da mortalidade, embora estatisticamente não significante, nos 2 grupos que receberam as maiores doses de Oxygent, sugere que a droga tenha, provavelmente, favorecido a oxigenação miocárdica, conjectura baseada em outros achados experimentais relativos aos efeitos deletéreos da hemodiluição sobre o metabolismo e função do coração $3,11,28$.

\section{CONCLUSÕES}

Em contraposição à hipótese investigada, o Oxygent não aumentou significativamente $\circ \mathrm{C}_{\mathrm{a}} \mathrm{O}_{2}$ nem, conseqüentemente, a $\mathrm{DO}_{2}$, muito embora 0 grupo com maior fluorocrito (Grupo 4) tenha apresentado $\mathrm{C}_{\mathrm{a}} \mathrm{O}_{2}$ consistentemente mais elevado que o dos demais. Isto sugere que doses ainda maiores da emulsão possam vir a promover aumentos significativos de $\mathrm{C}_{\mathrm{a}} \mathrm{O}_{2}$. O Grupo 4 também apresentou significativa elevação da $\mathrm{P}_{v} \mathrm{O}_{2}$ em função do fluxo de perfusão e notou-se, ainda, conquanto sem atingir significação estatística, menor mortalidade nos 2 grupos traitados com as maiores doses do produto.

Este conjunto de informaçōes denota um efeito benéfico, dose-dependente, do Oxygent sobre o metabolismo do oxigênio, de importante significado clínico, cujo mecanismo permanece especulativo. 
VICENTE, W. V. A.; HOLMAN, W. L.; SPRUELL, R. D.; FERGUSON, E. R.; CLYMER, J. J.; MURRAH, C. P.; PACIFICO, A. D. Emprego de uma nova emulsāo de perfluorocarbono (Oxygent MR) em circulaçăo extracorpórea: estudo experimental em cães. Rev. Bras. Cir. Cardiovasc., 10 (1): 34-42, 1995.

RBCCV 44205-256

VICENTE, W. V. A.; HOLMAN, W. L.; SPRUELL, R. D.; FERGUSON, E. R.; CLYMER, J. J.; MURRAH, C. P.; PACIFICO, A. D. - Use of a new perfluorocarbon emulsion (Oxygent ${ }^{T M}$ ) in a profoundly anemic canine model of hypothermic cardiopulmonary bypass. Rev. Bras. Cir. Cardiovasc., 10 (1): 34-42, 1995.

ABSTRACT: A novel perfluorocarbon emulsion (Oxygent ${ }^{T M}$ - Alliance Pharmaceutical Corp., San Diego, CA 92121, USA) was tested during hypothermic $\left(32^{\circ} \mathrm{C}\right)$ cardiopulmonary bypass $(\mathrm{CPB})$ under severe hemodilution ( $\mathrm{Hto}=12 \%)$. Forty-two mongrel dogs were allocated to 4 groups. Group $1(n=12)$ was not given Oxygent and served as control whereasGroups 2,3, and 4 ( 10 animals each) were given a bolus dose of the emulsion (1.5, 3.0, and $6.0 \mathrm{ml} / \mathrm{kg}$, respectively) at the onset of CPB. $\mathrm{O}_{2}$ metabolism variables were then studied at 6 randomized pump flow rates $(Q)$ ranging from 0.25 to $3.0 \mathrm{l} / \mathrm{min} / \mathrm{m}^{2}$. After rewarming CPB was discontinued and the animals were followed-up for one hour and then killed by IV KCl injection. Differences between groups were evaluated by the least squares means and Duncan's tests and considered significant for $p<0.05$. Fluorocrit values remained stable on CPB Group 2 - $1 \%$, Group $3-2 \%$, and Group $4-3 \%$ ). Although somewhat elevated, Group 4 arterial blood $\mathrm{O}_{2}$ partial pressure and content $\left(\mathrm{C}_{\mathrm{a}} \mathrm{O}_{2}\right)$ didn't reach statistical significance versus control.Group 4 mixed venous blood $\mathrm{O}_{2}$ partial pressure $\left(\mathrm{PvO}_{2}\right)$ regressed against $\mathrm{Q}$ was significantly higher $(p<0.03)$ than control, but there was no statistical difference when this variable was regressed against $\mathrm{O}_{2}$ delivery rate. Groups were similar regarding total as well as dissolved $\mathrm{O}_{2}$ consumption and systemic and transmyocardial lactate gradients. A trend towards lower mortality rates was noticed in Groups $3(20 \%)$ and $4(10 \%)$ versus control $(41.6 \%)$. Group 4 trend towards both reduced mortality and higher $\mathrm{C}_{\mathrm{a}} \mathrm{O}_{2}$ as well as its significant $\mathrm{PvO}_{2}$ rise against $\mathrm{Q}$ suggest a beneficial, yet speculative, Oxygent dose dependent effect on $\mathrm{O}_{2}$ metabolism.

DESCRIPTORS: Perfluorocarbons, in extracorporeal circulation. Oxygen metabolism. Extracorporeal circulation.

AGRADECIMENTOS: Aos Srs. Ronnie Brown, Jefferson Dudleston e Fred Wallace, pela inestimável assistência técnica. À Sras. Laucéa Conrado S. Porciúncula e Marlene Lúcio, pela edição do texto. Ao CNPq, pela bolsa de estudos.

\section{REFERÊNCIAS BIBLIOGRÁFICAS}

1 ALLIANCE PHARMACEUTICAL CORPORATION. Cardiovascular Pharmacology Division. Technical information. 1993.

2 BIRO, G. P. \& BLAIS, P. - Perfluorocarbon blood substitutes. Crit. Rev. Oncol. Hemat., 6: 311-374, 1987.

3 BRAZIER, J.; COOPER, N.; MALONEY Jr., J. V.; BUCKBERG, G. - The adequacy of myocardial oxygen delivery in acute normovolemic anemia. Surgery, 75, 508-516, 1974.

4 CONFORTO, A. B. \& FOCESI Jr., A. - Soluçōes artificiais e de hemoglobina como substitutos de sangue: uma revisão. Medicina (Ribeirão Preto), 24: 41-52, 1991.

5 ENGELMAN, R. M.; ROUSOU, J. H.; DOBBS, W. A. Fluosol-DA: an artificial blood for total cardiopulmonary bypass. Ann. Thorac. Surg., 32: 528-535, 1981.
6 FAITHFULL, N. S. \& CAIN, S. M. - Critical levels of $\mathrm{O}_{2}$ extraction following hemodilution with dextran and Fluosol-DA. J. Crit. Care, 3: 14-18, 1988.

7 FAITHFULL, N. S.; ERDMANN, W.; FENNEMA, M.; KOK, A. - Effects of haemodilution with fluorocarbons or dextran on oxygen tensions in the acutely ischaemic myocardium. Br. J. Anaesth., 58: 1031-1040, 1986.

8 FEOLA, M.; SIMONI, J.; ANGELILLO, R.; LUHRUMA, Z.; KABAKELE, M.; MANZOMBI, M.; KALUILA, M. Clinical trial of a hemoglobin based blood substitute in patients with sickle cell anemia. Surg. Gynecol. Obstet., 174: 379-386, 1992.

9 FERGUSON, E. R.; CLYMER, J. J.; SPRUELL, R. D.; HOLMAN, W. L. - Perfluorocarbon oxygen transport: a comparative study of four oxygenator designs. Surg. Forum, 1994. (No prelo).

10 FOX, L. S.; BLACKSTONE, E. H.; KIRKLIN, J. W. STEWART, R. W.; SAMUELSON, P. N. - Relationship of whole body oxygen consumption to perfusion flow rate during hypothermic cardioplegic cardiopulmonary bypass. J. Thorac. Cardiovasc. Surg., 83: 239-248, 1982.

11 HEIMISCH, S. H.; MEISNER, H.; ERBEN, R.; BAUM, M.; MENDLER, N. - The effect of hemodilution on regional myocardial function in the presence of coronary stenosis. Basic Res. Cardiol., 72: 344$364,1977$. 
VICENTE, W. V. A.; HOLMAN, W. L.; SPRUELL, R. D.; FERGUSON, E. R.; CLYMER, J. J.; MURRAH, C. P.; PACIFICO, A. D. Emprego de uma nova emulsăo de perfluorocarbono (Oxygent MR) em circulaçăo extracorpórea: estudo experimental em cães. Rev. Bras. Cir. Cardiovasc., 10 (1): 34-42, 1995.

HOLMAN, W. L.; MCGIFFIN, D. C.; VICENTE, W. V. A.; SPRUELL, R. D.; PACIFICO, A. D. - Use of current generation perfluorocarbon emulsions in cardiac surgery. Biomat. Art. Cells Immob. Biotech. (No prelo).

HOLMAN, W. L.; SPRUELL, R. D.; DIGERNESS, S. B.; DUDELSTON, J.; PACIFICO, A. D. - Oxyhemoglobin dissociation during hypothermic blood cardioplegia arrest. Circulation, 86 (Supl.2): 339-345, 1992.

HOLMAN, W. L.; SPRUELL, R. D.; FERGUSON, E. R.; CLYMER, J. J.; VICENTE, W. V. A.; MURRAH, P.; PACIFICO, A. D. - Tissue oxygenation with graded dissolved oxygen delivery during cardiopulmonary bypass. J. Thorac. Cardiovasc. Surg. (Encaminhado para publicação).

HOMER, L. D.; WEATHERSBY, P. K.; KIESOW, L. A. - Oxygen gradients between red blood cells in the microcirculation. Microvasc. Res., 22: $308-323$, 1981.

HONIG, C. R.; CONNETT, R. J.; GAYESKI, T. E. J. Interaction of blood flow, diffusive transport and cell metabolism in isovolemic anemia. In: GOLDSTICK, T. K. (ed.) Oxygen transport to tissues - 13. New York, Plenum Press, 1992. p. 21-29.

17 KEIPERT, P. E.; FAITHFULL, S.; BRADLEY, J. D.; HAZARD, D. Y.; HOGAN, J.; LEVISETTI, M. S.; PETERS, R. M. - Oxygen delivery augmentation by low-dose perfluorochemical emulsion during profound normovolemic hemodilution. In: VAUPEL, P. (ed.) Oxygen transport to tissues-15, New York, Plenum Press. (No Prelo).

LONGMUIR, I. S. - The effect of hypothermia on the affinity of tissues for oxygen. Life Sciences, 7: 297$300,1962$.

19 LUGO, G.; ARIZPE, D.; DOMINGUEZ, G.; RAMIREZ J.; TAMARIZ, O. - Relationship between oxygen consumption and oxygen delivery during anesthesia in high-risk surgical patients. Crit. Care Med., 21: 64-69, 1993.

20 MEINERT, H.; FACKLER, R.; KNOBLICH, A.; MADER, J.; REUTER, P.; ROHLKE, W. - On the perfluorocarbon emulsions of second generation. Biomat. Art. Cells Immob. Biotech., 20: 805-818, 1992.

RIESS, J. G. - Fluorocarbon-based in vivo oxygen transport and delivery systems. Vox Sang., 61: 225239, 1991a.
RIESS, J. G. - Hemocompatible fluorocarbon emulsions. In: SHARMA, C. P. \& SZYCHER, M. (eds.) Blood compatible materials and devices: perspectives towards the 21st century., Lancaster, Technomic Publishing Company, Inc., 1991b, cap. 14, p. 237 270.

23 RIESS, J. G.; DALFORS, G. K.; HANNA, G. K.; KRAFFT, M. P.; PELURA, T. J.; SCHUTT, E. G. - Development of highly fluid, concentrated and stable fluorocarbon emusions for diagnosis and therapy. Biomat. Art. Cells. Immob. Biotech., 20: 839-842, 1992.

24 ROUSOU, J. A.; ENGELMAN, R. M.; ANISIMOWICZ, L.; DOBBS, W. A. - A comparison of blood and Fluosol-DA for cardiopulmonary bypass. J. Cardiovasc. Surg., 26: 447-453, 1985.

25 SAMSEL, R. W. \& SCHUMACHER, P. T. - Determination of critical $\mathrm{O}_{2}$ delivery from experimental data: sensitivity to error. J. Appl. Physiol., 64: 2074-2082, 1988.

26 SCHOLANDER, P. F. - Oxygen transport through hemoglobin solutions. Science, 131: 585-590, 1960.

27 SIDI, A. \& DAVIS, R. F. - Lactate extraction fails to accurately reflect regional lactate production in ischemic myocardium. J. Cardiothorac. Anesth., 3: 312-318, 1989.

28 SPAHN, D. R.; SMITH, R.; VERONEE, C. D.; MCRAE, R. L.; WEI-CHIH, H.; MENIUS, A. J.; LOWE, J. E.; LEONE, B. J. - Acute isovolemic hemodiltion and blood transfusion. J. Thorac. Cardiovasc. Surg., 105: 694-704, 1993.

29 SPENCE, R. K.; CARSON, J.; CERNAIANU, A. C.; DELROSSI, A. J. - Transfusion and surgery. Curr. Probl. Surg., 30: 1105-1180, 1993.

30 STONE, J. J.; PICCIONE Jr., W.; BERRIZBEITIA, L. D.; DANCE, G. R.; SCHOEN, F. J.; SHEMIN, R. J.; COHN, L. H. - Hemodynamic, metabolic, and morphological effects of cardiopulmonary bypass with a fluorocarbon priming solution. Ann. Thorac. Surg., 41: $419-424,1986$.

31 TUPPURAINEM, T.; SETTERGREN, G.; STENSVED, $P$. - The effect of arterial $\mathrm{pH}$ on whole body oxygen uptake during hypothermic cardiopulmonary bypass in man. J. Thorac. Cardiovasc. Surg., 98: 769-773, 1989.

32 ZAKHAROV, V. N. - Structural analysis of moving blood from the viewpoint of new principles of circulation mechanics. J. Cardiovasc. Surg., 35: 19-26, 1994. 